\title{
Algorithm to Calculate a Large Number of Roots of the Cross-Product of Bessel Functions
}

\author{
Edén Sorolla, Juan R. Mosig, Fellow, IEEE, and Michael Mattes, Member, IEEE
}

\begin{abstract}
This paper describes an algorithm to calculate a large number of roots of the cross-product of Bessel functions and of their first derivatives. The algorithm initially finds the roots of the zeroth order using an auxiliary function that exhibits the same roots as the original cross-products but with better behavior for numerical root search with the Newton-Raphson algorithm. In order to find the roots for higher orders, the algorithm follows a pyramidal scheme using the interlacing property of the cross-product of Bessel functions. The algorithm shows globally convergent behavior for a large range of values of the argument and of the order of the Bessel functions. The roots can be computed to any precision, limited only by the computer implementation, and the convergence is attained in six iterations per root in average, showing a much better performance than previous works for the calculation of these roots.
\end{abstract}

Index Terms-Algorithm, Bessel functions, cross-product, interlacing properties, McMahon's expansion, Newton-Raphson.

\section{INTRODUCTION}

$\mathbf{T}$ HE TWO cross-product combinations of Bessel functions and their derivatives appear frequently in physical problems having cylindrical [1] or spherical [2] symmetry. The roots of the cross-product of Bessel functions and their derivatives represent the eigenvalue solutions of problems like the accurate simulation of the behavior of discontinuities involving coaxial geometries. This is a basic staple in modern computer-aided design (CAD) tools for designing waveguide networks [3]-[5]. The computation of the radiated field produced by an openended coaxial probe, a very useful tool in electromagnetic diagnosis, also needs the calculation of these roots [6]-[11]. Finally, the representation of the electromagnetic field within curved waveguides [12], as well as the prediction of the radiated power spectrum of charged particles within accelerating cavities [13], also require the efficient and fast calculation of these roots.

Many studies have been realized to calculate the roots of these functions [14]-[20], but they have not provided a globally convergent algorithm or, in the best cases, they have found solutions only valid for certain extreme values of the coaxial cable aspect ratio $q$, or the order of the involved Bessel functions, $\nu$.

This paper refines the work presented in [21], decreasing significantly the amount of iterations needed to find the first root for $\nu=1$ of the TE mode with respect to the previous algorithm.

Manuscript received June 14, 2012; revised September 19, 2012; accepted November 09, 2012. Date of publication December 04, 2012; date of current version April 03, 2013.

The authors are with the Laboratoire d'Electromagnétisme et d'Acoustique, (LEMA), Ecole Polytechnique Fédérale de Lausanne (EPFL), 1015 Lausanne, Switzerland (e-mail: eden.sorolla@epfl.ch).

Color versions of one or more of the figures in this paper are available online at http://ieeexplore.ieee.org.

Digital Object Identifier 10.1109/TAP.2012.2231929
Moreover, the new algorithm extends the range of $q$ for which the algorithm converges by optimizing the position of the seed in the root search within the boundaries of the search intervals accordingly to $q$. The bounds of $q$ that ensure the convergence are determined by the numerical limits of floating-number implementation. In the previous work, approximately 40500 and 14000 roots per second were calculated for the limiting values $q=1.01$ and $q=100$, respectively, whereas the new algorithm finds around 65000 and 16000 roots per second for the same values of $q$. Nevertheless, the most interesting feature of the current algorithm is the capability of calculating the roots for higher and lower values of the coaxial cable aspect ratio since the present work is able to find the roots of the cross-product of Bessel function for $q=1.001$ and $q=1000$, showing a speed of 81000 and around 16000 roots per second, respectively, all calculated on a current desktop computer (Intel 4-cores $2.4 \mathrm{GHz}$ ). Furthermore, deeper explanation of the improved algorithm with the detailed steps to follow depicted in pseudocode is also provided in this paper.

\section{A. Outline}

The motivation to look for the roots of the cross-product of Bessel functions is shown in Section II, where the general solution of the Helmholtz equation in cylindrical coordinates for homogeneous boundary conditions and the algorithm to calculate the eigenvalues that satisfy them are shown. Section II-A explains the root search for the zero-order cross-product, which serves as basis to calculate the roots for the remaining orders. Section II-B shows how to calculate the roots for orders higher than zero, and Section II-C how to calculate the roots for the cross-product of the derivatives of Bessel function for any order. The descriptions of the algorithms are provided in pseudocode format. Section III shows the main results obtained for two extreme values of the parameter $q$ to show the reliability and robustness of the algorithm. The convergence speed, translated as the number of iterations needed to reach the desired convergence in the search of each root, is also presented at the end of this section. Finally, Section IV concludes the paper, analyzing the performance of the algorithm.

\section{THEORY}

The solution of the Helmholtz equation in cylindrical coordinates provides the electromagnetic potentials of an electromagnetic wave traveling within a certain medium with cylindrical symmetry like a coaxial waveguide [1], where $k$ is the wavenumber of the electromagnetic wave of frequency $\omega=$ $k \sqrt{\mu \epsilon}$. The constants $\mu$ and $\epsilon$ are the magnetic permeability and the electric permittivity, respectively, of the medium through which the wave propagates. 
The electromagnetic potentials, represented by $\Phi(\rho, \phi, z)$, where $\rho, \phi$, and $z$ define the cylindrical coordinates system, can be found by applying the method of the separation of variables to the Helmholtz equation, defining three ordinary differential equations, one for each coordinate. In order to ensure that the solution of the azimuthal equation, $Q_{\nu}(\phi)$, is single-valued, the separation constant $\nu$, what counts for the azimuthal frequency of the solution, must be an integer. For problems whose domain do not include the point $\rho=0$, the two linearly independent solutions of the radial equation are proportional to the Bessel functions of the first and the second kind, $J_{\nu}(\lambda \rho)$ and $Y_{\nu}(\lambda \rho)$, respectively, where $\nu$ is the order of the Bessel functions and $\lambda \in \mathbb{R}^{+}$represents the radial frequency of the solution. In the context of a coaxial waveguide, $a$ and $b$ are the diameter of the inner and outer conductor $(b>a>0)$, respectively, and $q$ represents the outer-to-inner-radius ratio, that is $q=b / a$.

Imposing Dirichlet or Neumann boundary conditions to calculate the proportionality factors of the radial solution leads to an homogeneous system of equations whose determinant must vanish in order to find the nontrivial results. The values of $\lambda$ that render the determinant zero constitute all the physically meaningful values of the radial frequency that ensures that the solution verifies the boundary conditions. Thus, if we make the change of variable $x=\lambda a$, and we define the ratio $q \equiv b / a$, the roots of the functions

$$
\begin{aligned}
& f_{\nu}(q, x) \equiv J_{\nu}(q x) Y_{\nu}(x)-J_{\nu}(x) Y_{\nu}(q x) \\
& \tilde{f}_{\nu}(q, x) \equiv J_{\nu}^{\prime}(q x) Y_{\nu}^{\prime}(x)-J_{\nu}^{\prime}(x) Y_{\nu}^{\prime}(q x)
\end{aligned}
$$

provide us with the general solution of the Dirichlet and the Neumann boundary-value problem, respectively. As the general solution is a linear combination of the particular solutions associated to the roots of the equations above, the TM and the TE modes of an electromagnetic wave propagating within a coaxial waveguide are characterized by

$$
\begin{aligned}
& \Phi^{D}(\rho, \phi, z) \\
& =\sum_{\nu=0}^{\infty} \sum_{s=1}^{\infty}\left[A_{\nu}^{(s)} J_{\nu}\left(y_{\nu}^{(s)}\right)+B_{\nu}^{(s)} Y_{\nu}\left(y_{\nu}^{(s)}\right)\right] Q_{\nu}(\phi) Z_{\nu}^{(s)}(z) \\
& \Phi^{N}(\rho, \phi, z) \\
& =\sum_{\nu=0}^{\infty} \sum_{s=1}^{\infty}\left[\tilde{A}_{\nu}^{(s)} J_{\nu}\left(\tilde{y}_{\nu}^{(s)}\right)+\tilde{B}_{\nu}^{(s)} Y_{\nu}\left(\tilde{y}_{\nu}^{(s)}\right)\right] Q_{\nu}(\phi) Z_{\nu}^{(s)}(z)
\end{aligned}
$$

where $s$ is the index of the root, $Z(z)$ is the solution of the longitudinal equation, and $\left(A_{\nu}^{(s)}, B_{\nu}^{(s)}\right)$ and $\left(\tilde{A}_{\nu}^{(s)}, \tilde{B}_{\nu}^{(s)}\right)$ are the proportionality factors of the radial solution for the TM and the TE modes, respectively. $J_{\nu}^{\prime}(x)$ and $Y_{\nu}^{\prime}(x)$ are the first derivatives of the Bessel functions with respect to $x$. On the other hand, the roots are normalized by defining $y_{\nu}^{(s)}=(\rho / a) c_{\nu}^{(s)}$, $\tilde{y}_{\nu}^{(s)}=(\rho / a) \tilde{c}_{\nu}^{(s)}$, where $c_{\nu}^{(s)}$ and $\tilde{c}_{\nu}^{(s)}$ are the roots of $f_{\nu}(q, x)$ and $\tilde{f}_{\nu}(q, x)$, respectively.

From the numerical calculation point of view, the series in (2) and (3) must be truncated, thus a certain number of roots $(s=$ $\left.s_{\max }\right)$ for $\nu=0$ and a certain maximum index $\left(\nu=\nu_{\max }\right)$ will be needed to find $\Phi(\rho, \phi, z)$ to the desired accuracy. As it will be shown later, the roots of $f_{\nu-1}(q, x)$ are needed to obtain the ones of $f_{\nu}(q, x)$. Therefore, the roots for $\nu=0$ are calculated first, and the rest of roots are found in ascending order of $\nu$, defining an inverted pyramidal scheme where the first roots are shown on the top (the same holds for the roots of $\tilde{f}_{\nu}(q, x)$ )

$$
\left\{\begin{array}{l}
c_{0}^{(1)}, c_{0}^{(2)}, \ldots, c_{0}^{\left(s_{\max }-\nu_{\max }\right)}, \ldots, c_{0}^{\left(s_{\max }-1\right)}, c_{0}^{\left(s_{\max }\right)} \\
c_{1}^{(1)}, c_{1}^{(2)}, \ldots, c_{1}^{\left(s_{\max }-\nu_{\max }\right)}, \ldots, c_{1}^{\left(s_{\max }-1\right)} \\
\vdots \\
c_{\nu_{\max }}^{(1)}, c_{\nu_{\max }}^{(2)}, \ldots, c_{\nu_{\max }}^{\left(s_{\max }-\nu_{\max }\right)}
\end{array}\right\} .
$$

It is required that $s_{\max } \geq \nu_{\max }+1$ in order to calculate the last root of the scheme. Namely, if $p$ roots are requested for $\nu_{\max }=m$ to reach the desired accuracy in the calculation of $\Phi(\rho, \phi, z)$, the expression $s_{\max }=p+m$ must be satisfied.

\section{A. Roots of $f_{0}(q, x)-\mathrm{TM}_{0, s}$ Modes}

To calculate the first set of roots of $f_{0}(q, x)$ an auxiliary function is used following a similar approach as in [22]

$$
F_{0}(q, x) \equiv \frac{f_{0}(q, x)}{f_{0}^{\prime}(q, x)}
$$

since the roots of $f_{0}(q, x)$ and $f_{0}^{\prime}(q, x)$ never coincide [23]. Thus, the roots of $F_{0}(q, x)$ and $f_{0}(q, x)$ are the same, with the advantage that $F_{0}(q, x)$ shows a tangent-like function behavior as it is shown next. This guarantees the success of the Newton-Raphson (NR) algorithm [24] in the root search since all the approximations along the iterative process will lie inside the interval defined by the branches of the function $F_{0}(q, x)$.

Let us first calculate $F_{0}^{\prime}\left(q, x_{0}\right)$ when $F_{0}\left(q, x_{0}\right)=0$

$$
F_{0}^{\prime}\left(q, x_{0}\right)=\frac{\left(f_{0}^{\prime}\left(q, x_{0}\right)\right)^{2}-f_{0}\left(q, x_{0}\right) f_{0}^{\prime \prime}\left(q, x_{0}\right)}{\left(f_{0}^{\prime}\left(q, x_{0}\right)\right)^{2}}=1
$$

since $f_{0}\left(q, x_{0}\right)=0$ in this case.

Let $z_{0}^{(s)}$ be the positive roots of $f_{0}^{\prime}(q, x)$ i.e., the extremes of $f_{0}(q, x)$

$$
z_{0}^{(s)}=x \in(0, \infty) \mid f_{0}^{\prime}(q, x)=0
$$

and let us study the asymptotes of the auxiliary function $F_{0}(q, x)$ in these limits taking into account the periodic character of the function $f_{0}(q, x)[25]$

$$
\begin{aligned}
& \lim _{x \rightarrow z_{0}^{(s)}(-)} \frac{\operatorname{sgn}\left[f_{0}(q, x)\right]\left|f_{0}(q, x)\right|}{\operatorname{sgn}\left[f_{0}^{\prime}(q, x)\right]\left|f_{0}^{\prime}(q, x)\right|} \\
& =\left\{\begin{array}{l}
\frac{+\left(\lim _{x \rightarrow z_{0}^{(s)}(-)}\left|f_{0}(q, x)\right|\right.}{+\left(\lim _{x \rightarrow z_{0}^{(s)}(-)}\left|f_{0}^{\prime}(q, x)\right|\right)} \\
-\left(\lim _{x \rightarrow z_{0}^{(s)}(-)}\left|f_{0}(q, x)\right|\right) \\
-\left(\lim _{x \rightarrow z_{0}^{(s)}(-)}\left|f_{0}^{\prime}(q, x)\right|\right)
\end{array}\right\}=+\infty .
\end{aligned}
$$




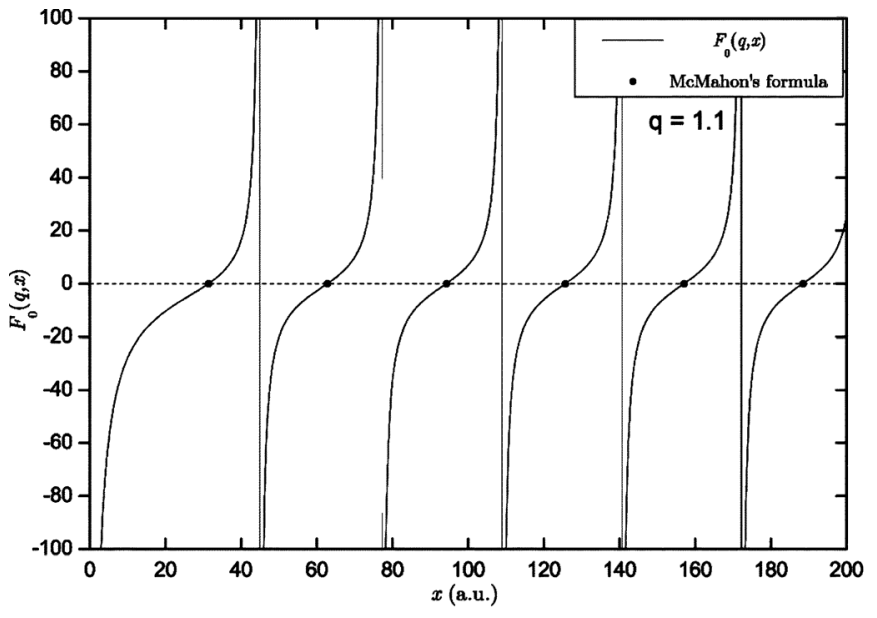

(a)

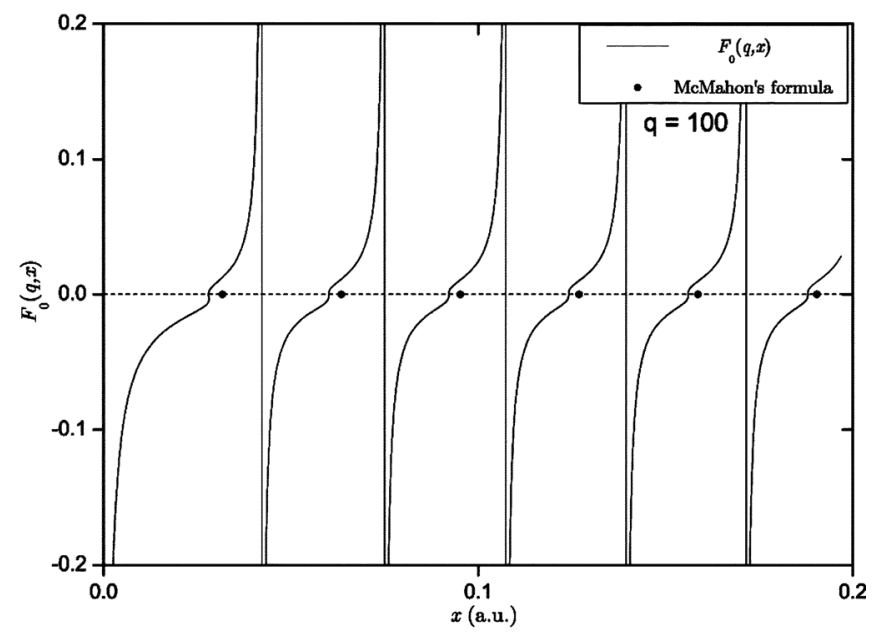

(b)

Fig. 1. Auxiliary function (solid line) and McMahon's expansion formula (dots) for the roots of (1a) for $\nu=0$. (a) $q=1.1$. (b) $q=100$.

The limits from the other side are

$$
\begin{aligned}
& \lim _{x \rightarrow z_{0}^{(s)}(+)} \frac{\operatorname{sgn}\left[f_{0}(q, x)\right]\left|f_{0}(q, x)\right|}{\operatorname{sgn}\left[f_{0}^{\prime}(q, x)\right]\left|f_{0}^{\prime}(q, x)\right|} \\
& =\left\{\begin{array}{l}
\left.\frac{+\left(\lim _{x \rightarrow z_{0}^{(s)}(+)}\left|f_{0}(q, x)\right|\right.}{-\left(\lim _{x \rightarrow z_{0}^{(s)}(+)}\left|f_{0}^{\prime}(q, x)\right|\right.}\right) \\
\left.\frac{-\left(\lim _{x \rightarrow z_{0}^{(s)}(+)}\left|f_{0}(q, x)\right|\right.}{+\left(\lim _{x \rightarrow z_{0}^{(s)}(+)}\left|f_{0}^{\prime}(q, x)\right|\right.}\right)
\end{array}\right\}=-\infty .
\end{aligned}
$$

Furthermore, due to the periodicity of $f_{0}(q, x)$, we know that there is a single root in between two extrema, thus between two roots of $f_{0}^{\prime}(q, x)$ there is a single zero of the function $F_{0}(q, x)$.

These properties show that $F_{0}$ resembles a tangent-like function. In Fig. 1, the auxiliary function $F_{0}(q, x)$ in solid line together with the seeds, represented by dots, used in the root search for $\nu=0$ is shown for two extreme values of the parameter $q$. The seeds of the root search are chosen accordingly to McMahon's expansion formula [14]. Note that the $s$ th seed lies within the branch corresponding to the same index of the root, and since McMahon's expansion formula provides a more precise approximation, the larger the value of the root, the consecutive seeds corresponding to larger values of $s$ are expected to lie within the corresponding branches as well. This guarantees the success of the root search using McMahon's expansion formula as seed for $\nu=0$.

The NR iteration formula needs the calculation of the first derivative of the auxiliary function. Therefore, recalling (4) and (5), we can write the root search formula as

$$
\begin{aligned}
X_{n+1} & =X_{n}-\frac{F_{0}\left(q, X_{n}\right)}{F_{0}^{\prime}\left(q, X_{n}\right)} \\
& =X_{n}-\left(\frac{f_{0}^{\prime}\left(q, X_{n}\right)}{f_{0}\left(q, X_{n}\right)}-\frac{f_{0}^{\prime \prime}\left(q, X_{n}\right)}{f_{0}^{\prime}\left(q, X_{n}\right)}\right)^{-1}
\end{aligned}
$$

where the prime indicates derivative with respect to $x$, with

$$
\begin{aligned}
f_{0}^{\prime}(q, x)= & {\left[J_{0}^{\prime}(q x) Y_{0}(x)-J_{0}(x) Y_{0}^{\prime}(q x)\right] } \\
& +q\left[J_{0}(q x) Y_{0}^{\prime}(x)-J_{0}^{\prime}(x) Y_{0}(q x)\right] \\
f_{0}^{\prime \prime}(q, x)= & A_{0}(x)+2 q B_{0}(x)+q^{2} C_{0}(x)
\end{aligned}
$$

and

$$
\left.\begin{array}{l}
A_{0}(x)=J_{0}^{\prime \prime}(q x) Y_{0}(x)-J_{0}(x) Y_{0}^{\prime \prime}(q x) \\
B_{0}(x)=J_{0}^{\prime}(q x) Y_{0}^{\prime}(x)-J_{0}^{\prime}(x) Y_{0}^{\prime}(q x) \\
C_{0}(x)=J_{0}(q x) Y_{0}^{\prime \prime}(x)-J_{0}^{\prime \prime}(x) Y_{0}(q x)
\end{array}\right\} .
$$

The algorithm to calculate the roots of $f_{0}(q, x)$ requests as input the ratio $q$, the desired maximum amount of roots $s_{\max }$ that are to be calculated for $\nu=0$, and the maximum order $\nu_{\max }$ considered for the rest of the root search, with $0<\nu_{\max }<s_{\max }$ (see pyramidal scheme). The desired accuracy and the maximum amount of iterations are given by eps and $i_{\max }$, respectively. The algorithm to obtain the set of roots for $\nu=0$ is then summarized in Algorithm 1.

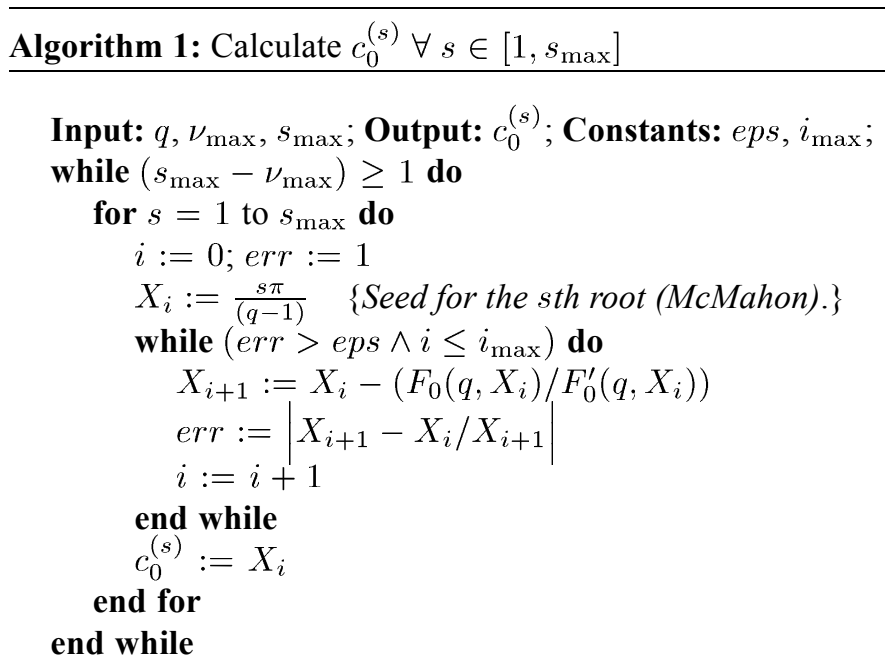

\section{B. Roots of $f_{\nu}(q, x)-\mathrm{TM}_{\nu, s}$ Modes}

It has been observed that the function $f_{\nu}(q, x)$ presents one and only one root in between two consecutive roots of $f_{\nu-1}(q, x)$ for any value of $q$ and $\nu$ [2]. These interlacing properties of the roots ensure the good performance of our algorithm since the roots of the set 


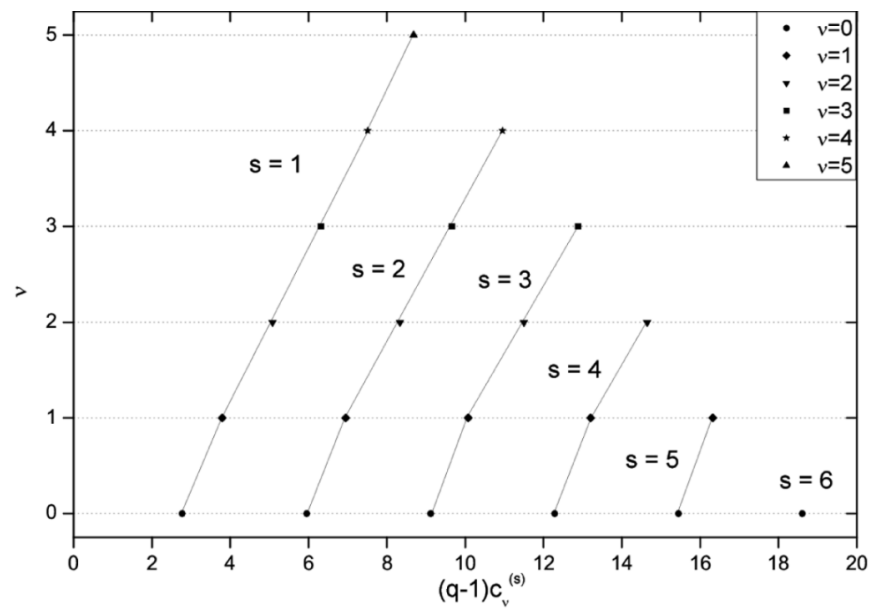

(a)

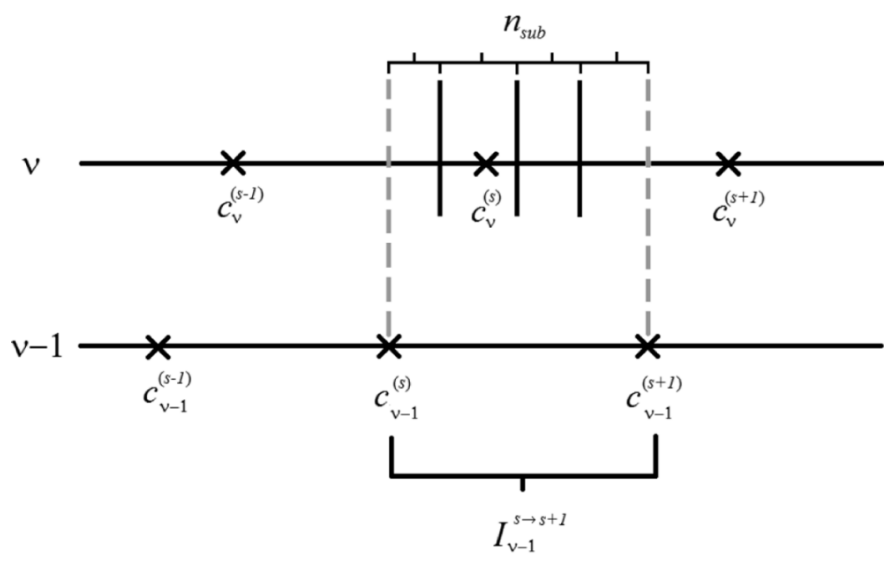

(b)

Fig. 2. (a) All the roots calculated by the algorithm for $s_{\max }=6, \nu_{\max }=5$, and $q=100$. (b) Sketch showing how the interval $I_{\nu-1}^{s \rightarrow+1}$ is split into an arbitrary number of subintervals $n_{\text {sub }}$.

$\mathcal{C}_{\nu}=\left\{c_{\nu}^{(s)} \mid f_{\nu}\left(q, c_{\nu}^{(s)}\right)=0, s \in \mathbb{N}, \nu \in \mathbb{N}^{0}\right\}$ are bounded by the roots of $\mathcal{C}_{\nu-1}$ as follows:

$$
c_{\nu-1}^{(s)}<c_{\nu}^{(s)}<c_{\nu-1}^{(s+1)} \quad \forall \nu>0 .
$$

Thus, to find the roots for $\nu>0$, the set $\mathcal{C}_{0}$ is used as a basic set from which the rest of the roots will be found using the original function $f_{\nu}(q, x)$. The interlacing properties can be observed in Fig. 2(a), where the roots of the function $f_{\nu}(q, x)$ for $q=100$ and different $\nu$ orders are shown. The search interval of each root for the order $\nu$ is therefore well established by the interlacing properties and defined by

$$
I_{\nu-1}^{s \rightarrow s+1} \in\left(c_{\nu-1}^{(s)}, c_{\nu-1}^{(s+1)}\right) \quad \forall \nu>0
$$

which is split into $n_{\text {sub }}$ subintervals [see Fig. 2(b)]. The seed is placed slightly above the beginning of the first subinterval by $\epsilon$, a quantity that is fixed by the maximum among the quantities: "machine precision times the interval length" and "machine precision." Therefore, the NR iteration is applied, and if some approximation to the root lies outside the interval, defined by the interlacing properties, the seed is placed at the beginning of the next subinterval and so on until the solution converges inside $I_{\nu-1}^{s \rightarrow s+1}$. The quasi-periodicity of the cross-product of Bessel functions [19] ensures that, placing the seed within some of the subintervals, the NR algorithm will necessarily converge. The procedure to calculate the roots for each order of the function $f_{\nu}(q, x)$, until $\nu=\nu_{\max }$ is reached, is shown in Algorithm 2.

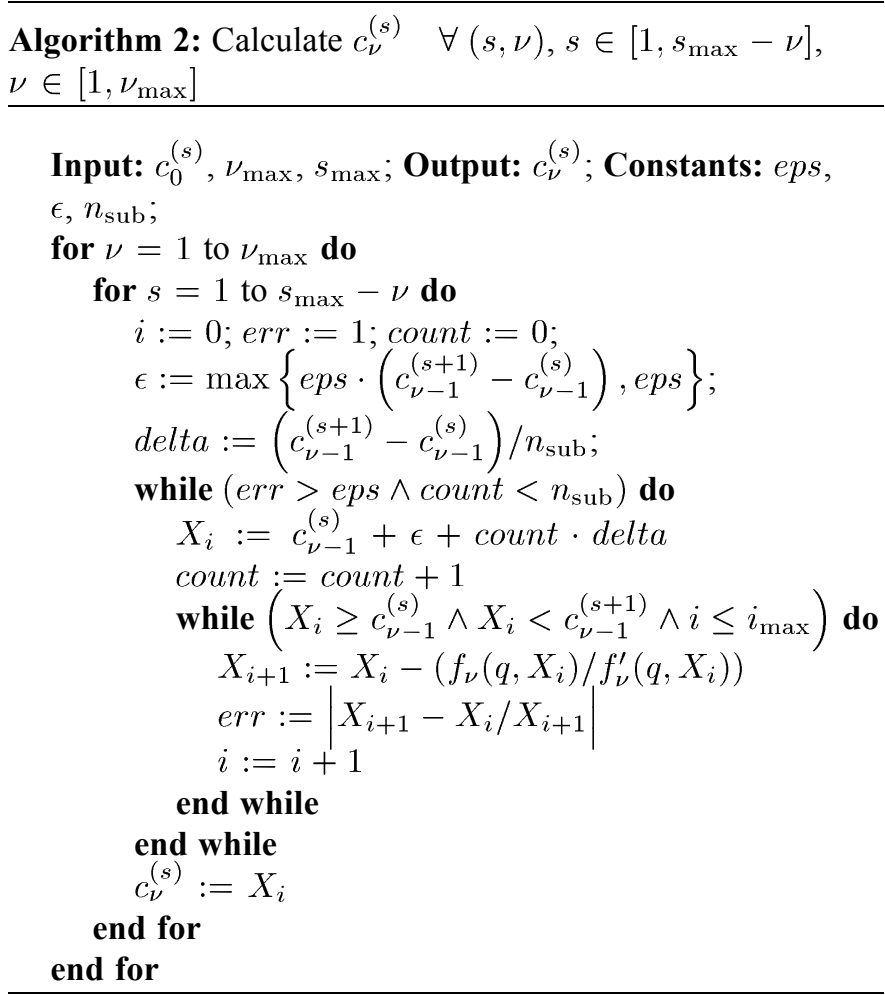

C. Roots of $\tilde{f}_{\nu}(q, x)-\mathrm{TE}_{\nu, s}$ Modes

To calculate the set of roots of (1b) for $\nu=0$, the property $\mathcal{Z}_{0}^{\prime}(x)=-\mathcal{Z}_{1}(x)$ [26] is used, where $\mathcal{Z}_{\nu}(x)$ is any of the two Bessel functions, either $J_{\nu}(x)$ or $Y_{\nu}(x)$. This property leads to $c_{1}^{(s)}=\tilde{c}_{0}^{(s)},(s=1,2,3, \ldots)$, thus the set of roots of $\tilde{f}_{\nu}(q, x)$ for $\nu=0$ is already given since it is the same as the set of roots of $f_{\nu}(q, x)$ for $\nu=1$, previously calculated. The interlacing properties

$$
\tilde{c}_{\nu-1}^{(s)}<\tilde{c}_{\nu}^{(s)}<\tilde{c}_{\nu-1}^{(s+1)} \quad \forall \nu>0
$$

are also valid for all the roots except for $\tilde{c}_{1}^{(1)}$ since it lies before $\tilde{c}_{0}^{(1)}$ [27]. This root is therefore searched inside the interval $\tilde{I}_{0} \in\left(\delta, \tilde{c}_{0}^{(1)}\right)$, where $\delta$ is an arbitrary number smaller than the root to be found, and $\tilde{c}_{0}^{(1)} \equiv c_{1}^{(1)}$ has been calculated previously. In the algorithm, it has been chosen $\delta=10^{-3}$ since the smallest root that can be found by our algorithm corresponds to $q=1000$ and, in this case, the searched root is around $1.8 \cdot 10^{-3}$. Once this root has been found, the remaining ones are automatically calculated following the same inverted pyramidal scheme and relying on the subdivision of the intervals performed by the roots of the previous $\nu$ order, as described above. Note that the index $s$ for the roots of $\tilde{f}_{\nu}(q, x)$ does not correspond to the one of McMahon's expansion since smaller roots than predicted for $s=1$ are found [27], [28]. Thus, the index $s$ is shifted by one in this case with respect to McMahon's nomenclature for the roots of this function.

The algorithm to calculate the set of roots $\tilde{\mathcal{C}}_{\nu}$ until reaching the order $\nu_{\max }$ is presented in Algorithm 3. 
TABLE I

RoOTS OF $f_{\nu}(q, x)$ FOR $q=1.001$

\begin{tabular}{|c|c|c|c|c|c|c|}
\hline \multicolumn{7}{|c|}{$(q-1) c_{\nu}^{(s)}$} \\
\hline $\mathrm{s}^{\nu}$ & 0 & 1 & 2 & 3 & 4 & 5 \\
\hline 1 & 3.14159261384070 & 3.14159277283666 & 3.14159324982459 & 3.14159404480405 & 3.14159515777465 & 3.14159658873654 \\
\hline 2 & 6.28318528730424 & 6.28318536680294 & 6.28318560529729 & 6.28318600278670 & 6.28318655927254 & 6.28318727475406 \\
\hline 3 & 9.42477794751978 & 9.42477800051970 & 9.42477815951523 & 9.42477842450749 & 9.42477879549898 & 9.42477927248715 \\
\hline 4 & 12.5663706044217 & 12.5663706441725 & 12.5663707634179 & 12.5663709621625 & 12.5663712404052 & 12.5663715981467 \\
\hline 5 & 15.7079632599985 & 15.7079632917983 & 15.7079633871946 & 15.7079635461920 & 15.7079637687865 & 15.7079640549799 \\
\hline 6 & 18.8495559149138 & 18.8495559414141 & 18.8495560209108 & 18.8495561534082 & 18.8495563389043 & 18.8495565773960 \\
\hline 7 & 21.9911485694487 & 21.9911485921646 & 21.9911486603049 & 21.9911487738743 & 21.9911489328691 & 21.9911491372929 \\
\hline 8 & 25.1327412237482 & 25.1327412436240 & 25.1327413032459 & 25.1327414026220 & 25.1327415417425 & 25.1327417206116 \\
\hline 9 & 28.2743338778918 & 28.2743338955588 & 28.2743339485548 & 28.2743340368878 & 28.2743341605521 & 28.2743343195481 \\
\hline 10 & 31.4159265319259 & 31.4159265478225 & 31.4159265955208 & 31.4159266750207 & 31.4159267863192 & 31.4159269294122 \\
\hline
\end{tabular}

TABLE II

ROOTS OF $\tilde{f}_{\nu}(q, x)$ FOR $q=1.001$

\begin{tabular}{|c|c|c|c|c|c|}
\hline \multicolumn{7}{|c|}{$(q-1) \tilde{c}_{\nu}^{(s)}$} \\
\hline $\mathrm{s}$ & 1 & 2 & 3 & 4 & 5 \\
\hline $1\left(10^{-3}\right)$ & 0.9995002914791958 & 1.999000582958197 & 2.998500874437121 & 3.998001165915639 & 4.9975014573938446 \\
\hline 2 & 3.14159293183281 & 3.14159340882063 & 3.14159420380024 & 3.14159531677170 & 3.14159674773360 \\
\hline 3 & 6.28318544630149 & 6.28318568479540 & 6.28318608228523 & 6.28318663877034 & 6.28318735425205 \\
\hline 4 & 9.42477805351680 & 9.42477821251291 & 9.424778477750602 & 9.42477884849725 & 9.42477932548451 \\
\hline 5 & 12.5663706839191 & 12.5663708031668 & 12.5663710019127 & 12.5663712801540 & 12.5663716378951 \\
\hline 6 & 15.7079633235970 & 15.7079634189947 & 15.7079635779910 & 15.7079638005860 & 15.7079640867774 \\
\hline 7 & 18.8495559679116 & 18.8495560474122 & 18.849556179968 & 18.8495563654032 & 18.8495566038958 \\
\hline 8 & 21.9911486148778 & 21.9911486830198 & 21.9911487965874 & 21.9911489555840 & 21.9911491600060 \\
\hline 9 & 25.1327412634983 & 25.1327413231240 & 25.1327414224935 & 25.1327415616179 & 25.1327417404876 \\
\hline 10 & 28.2743339132224 & 28.2743339662221 & 28.2743340545521 & 28.2743341782166 & 28.2743343372131 \\
\hline 11 & 31.4159265637216 & 31.4159266114206 & 31.4159266909218 & 31.4159268022147 & 31.4159269453126 \\
\hline
\end{tabular}

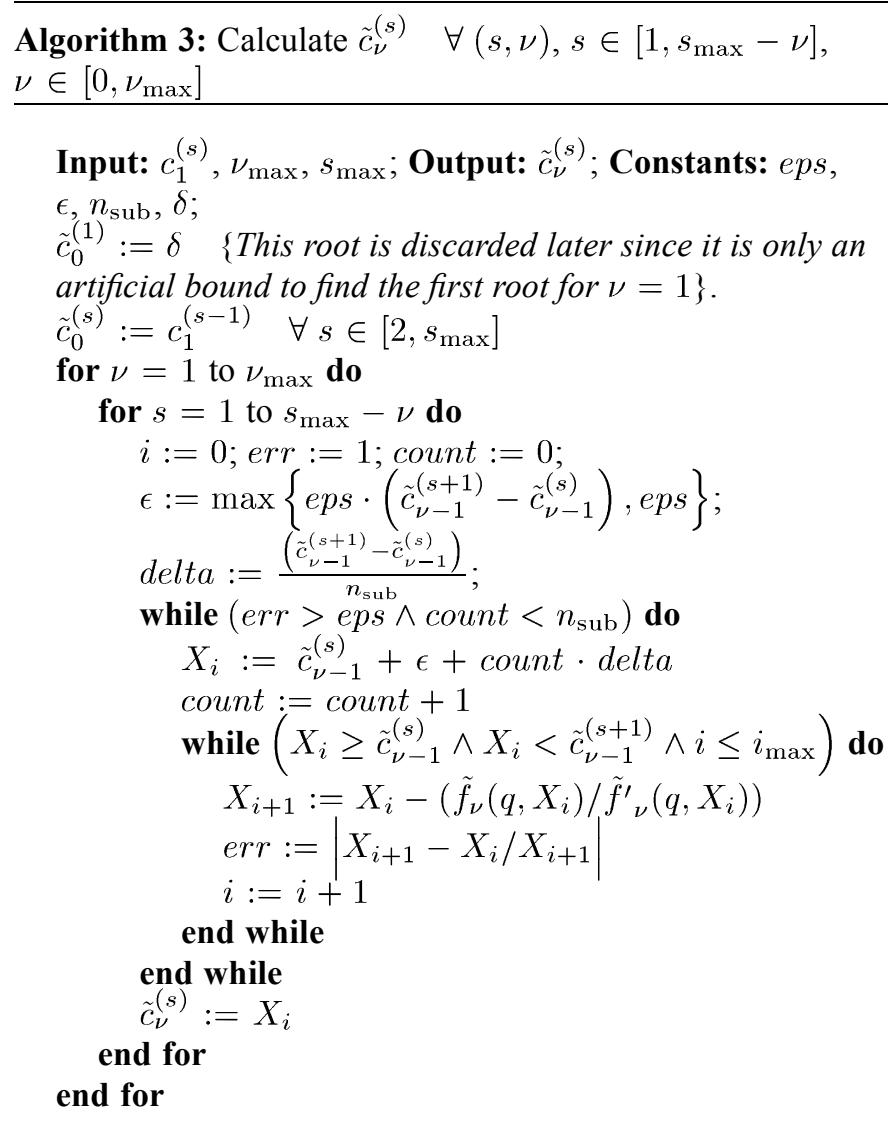

\section{MAIN RESUlts}

In this section, the roots of the two functions (1) are shown for two extreme values of the parameter $(q=1.001$ and $q=1000)$ in order to check the reliability of the algorithm for limiting cases. This ensures that for ratios of $q$ as there are found in practical applications, the algorithm will work as well. For these two extreme values, the interlacing properties of the roots of both the cross-product of Bessel functions and their derivatives are verified.

As for low values of $q$, the roots of (1) for a given $s$ lie very close to each other as $\nu$ increases, and the interlacing properties cannot be easily verified by visual inspection; we represent the roots for $q=1.001$ in tabular format normalized by the quantity $(q-1)$. In Table I, the 10 first roots of (1a) for values of $\nu$ from 0 to 5 are shown. In Table II, the 11 first roots of (1b) for values of $\nu$ from 1 to 5 for the same value of $q$ are shown. The reason why the roots in Table II for $\nu=0$ are not shown is because they are the same as those ones of Table I for $\nu=1$ as it has been pointed out before. It can be seen that the values of the normalized roots tend to multiples of $\pi$, as expected when $q \rightarrow 1$ since this limit corresponds to the solution of a parallel-plate waveguide [18]. The results certify that the interlacing properties of the roots of both functions hold for all of them.

For large values of $q$, the verification of the interlacing properties can be easily performed by visual inspection. The 10 first roots of the cross-product of Bessel functions and the ones of the cross-product of their derivatives are shown for the 10 first values of $\nu$ for $q=1000$ in Fig. 3(a) and (b), respectively. In 


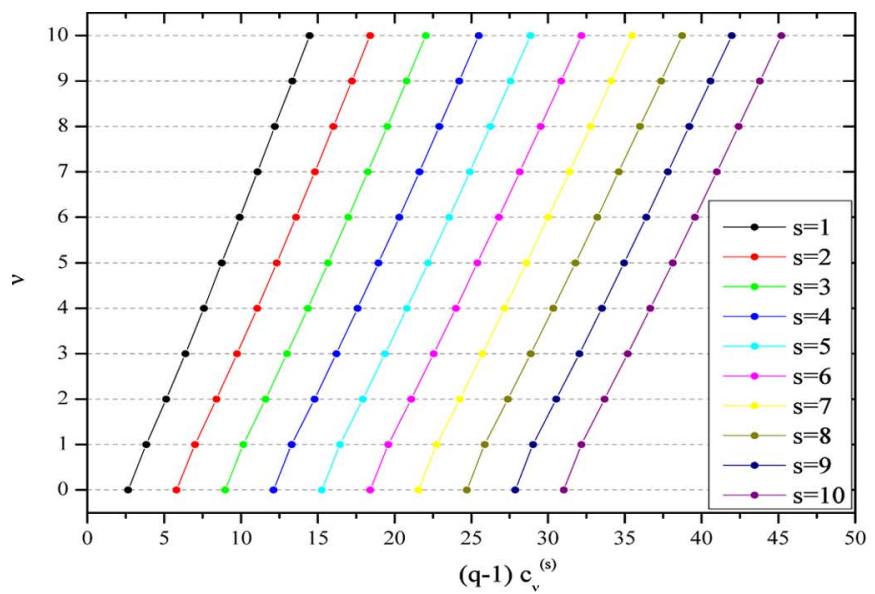

(a)

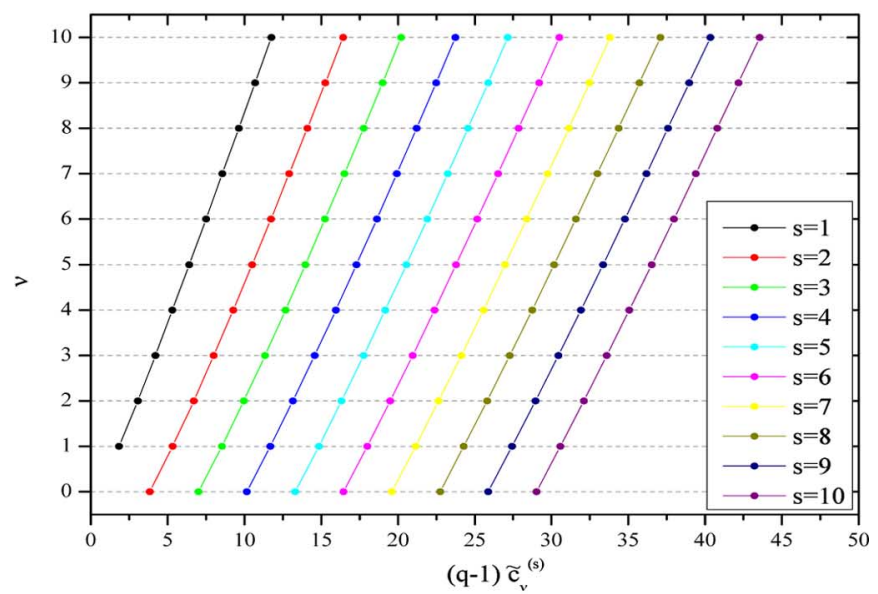

(b)

Fig. 3. Roots of (1) for $q=1000$. (a) Roots of $f_{\nu}(q, x)$. (b) Roots of $\hat{f}_{\nu}(q, x)$.

this case, it can be noticed that the roots of (1a) tend to the ones of the Bessel function of the first kind, that is, the roots of $J_{\nu}(q x)=0$, while the roots of $(1 \mathrm{~b})$ tend to the ones of the derivative $J_{\nu}^{\prime}(q x)=0$, as expected [29].

In order to show the efficiency of the roots search, the number of iterations needed to reach machine precision for the first root of the functions defined in (1) is represented in Fig. 4. The convergence study of the first root of $f_{\nu}$ [Fig. 4(a)] shows that the number of iterations needed to reach machine-precision accuracy increases as $\nu$ grows for a given $q$, though the convergence seems to depend more on $q$ than on the index of the Bessel functions. Namely, for a given $\nu$, the convergence is more quickly attained as the ratio $q$ decreases and gets close to one. This is due to the fact that the McMahon's expansion provides a better estimate of the roots as the ratio $s /(q-1)$ increases. Another reason to explain why the algorithm needs less iterations for lower values of $q$ than for larger ones is due to the fact that the algorithm places the seed slightly above the value of $c_{\nu-1}^{(s)}$ to find the root $c_{\nu}^{(s)}$. Thus, as the root lies closer to the seed for smaller values of $q$ than for larger ones (compare results in Table I to those of Fig. 3), the convergence is more quickly attained for $q \rightarrow 1$. The fast and accurate character of the algorithm to find all the roots of functions (1) for any $\nu$ is, indeed, based on the knowledge of the set $\mathcal{C}_{0}$ and the properties of its elements with respect to the roots for higher orders $\nu$. Nevertheless, the plot

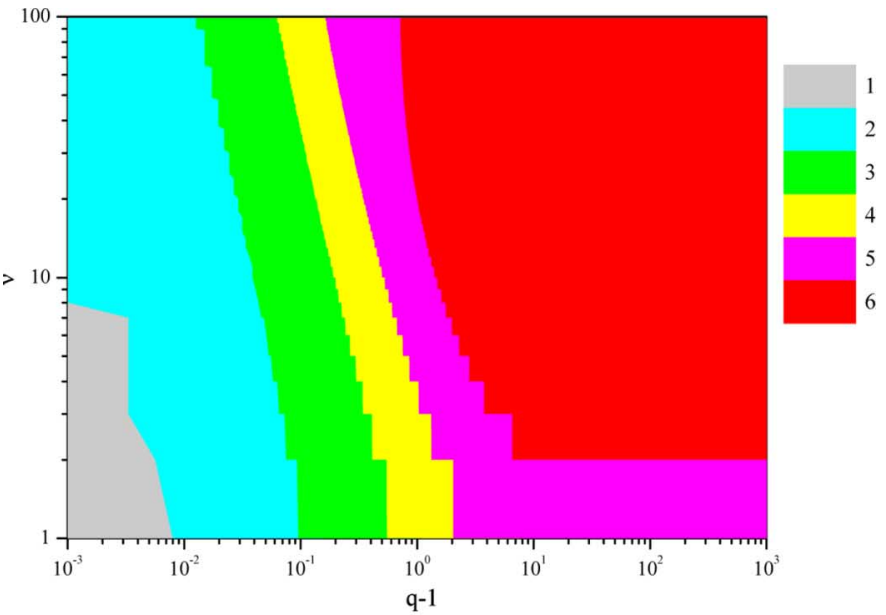

(a)

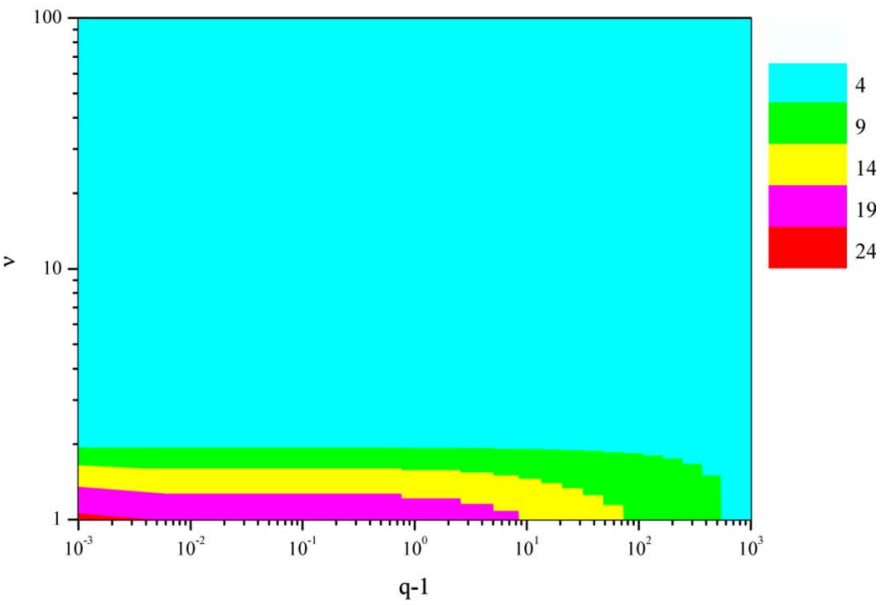

(b)

Fig. 4. Convergence study of (a) $c_{\nu}^{(1)}$ and (b) $\tilde{c}_{\nu}^{(1)}$ (bottom).

shows that in the worst case, corresponding to $(q-1)=1000$, the algorithm only needs six steps to reach machine-precision accuracy for $\nu=100$, which reflects the fast performance of the calculation.

It must be remarked that the occasional jump of the seed [cf. Fig. 2(b) and Section II-B] to the beginning of the second or the third subintervals when the approximation to the root lies outside the search interval has been recorded and taken into account in the plot of Fig. 4. The convergence study of the root search for $n_{\text {sub }}=10$ shows the presence of the jump of the seed to the second and the third subintervals for values of $q$ above 5 and 50, respectively. No jump of the seed beyond the third subinterval was observed for values of $q$ below 1000 when $\nu$ and $s$ range from 0 to 100 and from 1 to 100 , respectively. The appearance of jumps in the seed to the next subintervals does not follow a clear tendency with the parameter $q$ or the index $\nu$ since it depends on the slope of the function $f_{\nu}(q, x)$ (or $\tilde{f}_{\nu}(q, x)$ ) evaluated at $x=c_{\nu-1}^{(s)}$ (or $x=\tilde{c}_{\nu-1}^{(s)}$ ). If the absolute value of the slope is very small the NR iteration will approximate the root outside the search interval defined by $I_{\nu \rightarrow 1}^{s \rightarrow 1}$, but the periodicity of the cross-product of the Bessel functions ensures that the slope of the function evaluated at the beginning of some subinterval will guarantee the convergence of the root search.

For example, for $q=5$, jumps to the second subinterval were recorded for values of $\nu$ between 21 and 55 for a few values of 
the root index, $s$. For $\nu=21$ the jump of the root approximation to the second subinterval was observed only for $s=18,19$. For $\nu=55$, this jump occurred only for $s=44,45$. The behavior of the jump of the seed to the second subinterval for intermediate values of $\nu$ was different. For instance, for $\nu=41$, this occurred for the roots lying between $s=33$ and $s=43$.

For $q=1000$, the presence of the jumps of the seed to the next subintervals was observed for values of $\nu$ from 2 to 47 . For values of $\nu$ below 15, the jump to the third subinterval occurred in few cases for large values of the root index, $s$ (between 70 and 90). For big values of $\nu$, the jump occurred only to the second subinterval for a few roots (typically eight for each value of $\nu$ ). It has also been observed that the number of roots for which this jump occurs decreases as the index $\nu$ grows, no jumps occurring for $\nu>47$.

Fig. 4(b) shows that the convergence of the first root of $\tilde{f}_{\nu}$ is mainly achieved in four or less iterations for any value of $q$ as long as $\nu$ is larger than one, reflecting the satisfactory optimization of the method. It is worthwhile to mention that, unlike the convergence of the search of $c_{\nu}^{(1)}$, the number of iterations needed to find $\tilde{c}_{\nu}^{(1)}$ is practically independent of $q$, except for the first order, showing a satisfactorily performance of the root search for any order. The exception to the fast convergence of the algorithm occurs for $\nu=1$, where the algorithm needs up to 24 iterations to converge for $q=1.001$, though it is better compared to the previous algorithm [21], where 81 iterations were required for $q=1.01$. Although the comparison is made for different values of $q$ it must be noted that the lower the coaxial cable aspect ratio, the worse the convergence speed. The current algorithm finds the first root faster than the previous one and, moreover, for a lower value of $q$.

The reason for the slow performance in the root search of $\tilde{c}_{\nu}^{(1)}$ is twofold: The seed to calculate $\tilde{c}_{1}^{(1)}$ is placed slightly above $\delta$ within the interval $\tilde{I}_{0} \in\left(\delta, c_{1}^{(1)}\right)$, and this may lie far away from the actual root; the second reason is that the slope of the function $\tilde{f}_{1}(q, x)$ is very steep close to the origin, what makes the NR algorithm difficult to converge when the seed is far from the root. Let us recall that the lower bound within the search interval was optimized to lie close to the first root for $q=1000$ [see Fig. 5(b)], and the NR algorithm in this case needs only four steps as it is shown in cyan in Fig. 4(b). For lower values of $q$, the first root for $\nu=1$ lies far above the lower bound of the search interval, what slows down the convergence. Nevertheless, all the roots $\tilde{c}_{\nu}^{(s)}$ are calculated based on the knowledge of $\tilde{c}_{\nu-1}^{(s)}$. Therefore, the drawback found in the root search of the first root of $\tilde{f}_{1}(q, x)$ almost does not affect the whole performance of the algorithm since, once the value of $\tilde{c}_{1}^{(1)}$ is found, the algorithm calculates the rest of the roots very efficiently for any value of $\nu$ and $q$.

The average number of iterations needed to calculate the eigenvalues of the TM modes is around 6 per root for $q>3$ and around 2 for $q<1.1$. For the TE modes, the behavior of the convergence is in general a bit better, apart from the fact that for low values of $q$, the convergence for $\nu=1$ is very slow for the reasons mentioned above. In the end, the overall effect is that the average number of iterations in the search of the eigenvalues of TM and TE modes is very similar. The calculations of the roots have been carried out on a common desktop computer (Intel 4-cores $2.4 \mathrm{GHz}$ ), the algorithm showing a

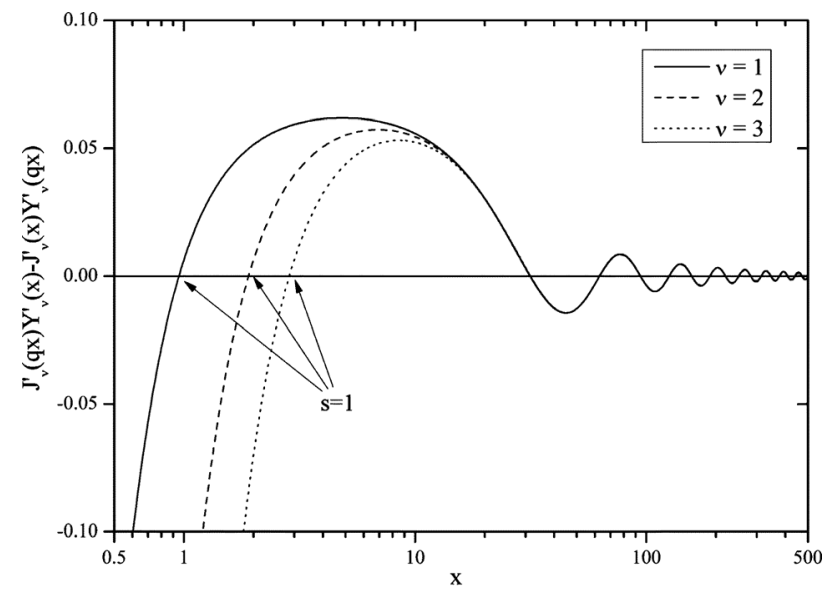

(a)

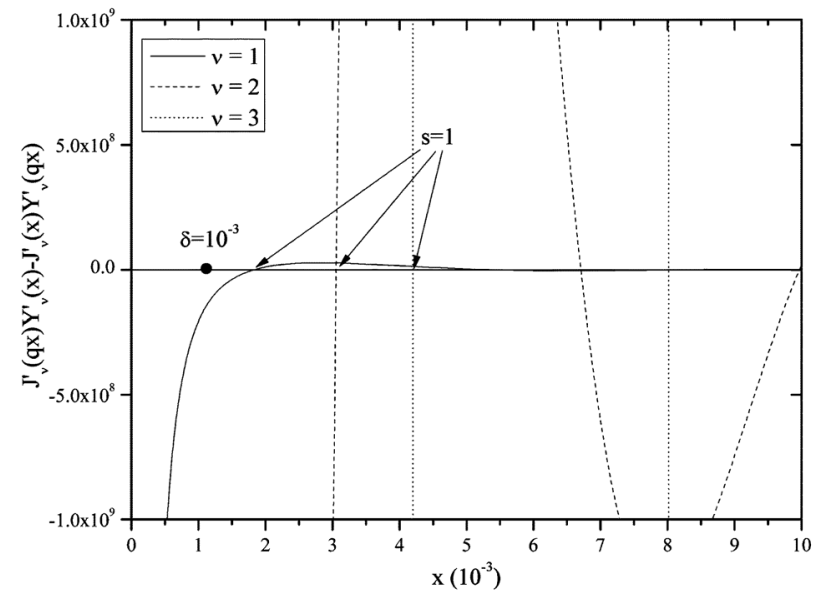

(b)

Fig. 5. Plot of the function $\tilde{f}_{\nu}(q, x)$. (a) $q=1.1$. (b) $q=1000$.

speed of around 81000 and around 16000 roots per second for $q=1.001$ and $q=1000$, respectively.

The previously developed algorithms that analyzed the calculation of the roots of (1) were based either on the approximation by a series [14], [15], sometimes only valid for asymptotic values of $q$ or $\nu$ [16], [18], or on the solution of an eigenvalue problem in a fast and efficient way as most recent works have done [3], [4], [30], [31]. These last studies showed very efficient calculation of the eigenvalues of more general structures, though the accuracy presented for coaxial cables was lower than achieved with the algorithm presented in this paper. Nevertheless, as the computational cost to solve a linear eigenvalue problem grows with the square of the amount of roots, the algorithm presented here is much more efficient when a large number of roots is requested since its computational cost grows only linearly.

\section{CONCLUSION}

A globally convergent algorithm to find the roots of the crossproduct of Bessel functions and their derivatives has been developed for the first time for a large range of values of the parameter $q$ and the order $\nu$. The algorithm initially finds the roots of the cross-product of Bessel functions for $\nu=0$ using an auxiliary function with the same roots. In order to find the roots for higher values of $\nu$, the algorithm follows a pyramidal scheme using the interlacing property of the cross-products. 
The efficiency of the method has been checked analyzing the amount of iterations needed to reach convergence up to the 14th digit, underlining its good performance. In average, approximately only six iterations per root are required considering two extreme values of the parameter $q$, with the exception of the first root of the cross-product of derivatives of Bessel functions. However, this isolated case only slightly affects the overall performance of the algorithm.

The speed of the algorithm has been tested for two extreme values of the parameter $q$ having been improved noticeably with respect to previous works together with the accuracy.

\section{REFERENCES}

[1] J. A. Stratton, Electromagnetic Theory. New York, NY, USA: McGraw-Hill, 1941.

[2] T. H. Boyer, "Concerning the zeros of some functions related to Bessel functions," J. Math. Phys., vol. 10, no. 9, pp. 1729-1744, 1969.

[3] B. Gimeno and M. Guglielmi, "Multimode equivalent network representation for junctions between coaxial and circular waveguides," Int. J. Microw. Millim.-Wave Comput.-Aided Eng., vol. 7, no. 2, pp. 180-194, 1996.

[4] B. Gimeno and M. Guglielmi, "Full wave network representation for rectangular, circular and elliptical to elliptical waveguide junctions," IEEE Trans. Microw. Theory Tech., vol. 45, no. 3, pp. 376-384, Mar. 1997.

[5] F. Arndt, "Fast CAD and optimization of waveguide components and aperture antennas by hybrid MM/FE/MoM/FD methods - State-ofthe-art and recent advances," IEEE Trans. Microw. Theory. Tech., vol. 52, no. 1, pp. 292-305, Jan. 2004.

[6] J. R. Mosig, J. C. E. Besson, M. Gex-Fabry, and F. E. Gardiol, "Reflection of an open-ended coaxial line and application to nondestructive measurement of materials," IEEE Trans. Instrum. Meas., vol. IM-30, no. 1, pp. 46-51, Mar. 1981.

[7] E. K. Yung, C. Eng, and C. M. Butler, "Coaxial-line driven monopole on an electrically thick conducting cylinder over a ground plane," Inst. Elect. Eng. Proc., vol. 131, no. 1, pp. 54-60, 1984.

[8] J. H. Lee, H. J. Eom, and K. H. Jun, "Reflection of a coaxial line radiating into a parallel plate," IEEE Microw. Guided Wave Lett., vol. 6, no. 3, pp. 135-137, Mar. 1996.

[9] Y. C. Noh and H. J. Eom, "Radiation from a flanged coaxial line into a dielectric slab," IEEE Trans. Microw. Theory Tech., vol. 47, no. 11, pp. 2158-2161, Nov. 1999.

[10] G. Panariello, L. Verolino, and G. Vitolo, "Efficient and accurate fullwave analysis of the open-ended coaxial cable," IEEE Trans. Microw. Theory Tech., vol. 49, no. 7, pp. 1304-1309, Jul. 2001.

[11] J. S. Asvestas, "Radiation of a coaxial line into a half-space," IEEE Trans. Antennas Propag., vol. 54, no. 6, pp. 1624-1631, Jun. 2006.

[12] I. V. Petrusenko, "High-order approximation of wave propagation constants in uniformly curved waveguide," Inst. Elect. Eng. Proc., Microw. Antennas Propag., vol. 148, no. 5, pp. 280-284, 2001.

[13] S. K. Nagesh, D. Revannasiddiah, and S. V. K. Shastry, "Investigation of multipactor breakdown in communication satellite microwave co-axial systems," Pramana, J. Phys., vol. 64, no. 1, pp. 95-110, 2005.

[14] J. McMahon, "On the roots of the Bessel and certain related functions," Ann. Math., vol. 9, no. 1/6, pp. 23-30, 1894.

[15] J. F. Bridge and S. W. Angrist, "An extended table of roots of $J_{n}^{\prime}(x) Y_{n}^{\prime}(\beta x)-J_{n}^{\prime}(\beta x) Y_{n}^{\prime}(x)=0$," Math. Comput., vol. 16, no. 78, pp. 198-204, 1962.

[16] L. J. Laslett and W. Lewish, "Evaluation of the zeros of cross-product Bessel functions," Math. Comput., vol. 16, pp. 226-232, 1962.

[17] H. Bauer, "Tables of zeros of cross product Bessel functions," Math. Comput., vol. 18, no. 85, pp. 128-135, 1964.

[18] J. A. Cochran, "The asymptotic nature of zeros of cross-product Bessel functions," Quart. J. Mech. Appl. Math., vol. 19, pp. 511-522, 1966.

[19] L. Z. Salchev and V. B. Popov, "Determination of the zeros of a crossproduct Bessel function," Proc. Camb. Phil. Soc., vol. 74, pp. 477-483, 1973.

[20] R. Koss, P. Crespo-Valero, and J. R. Mosig, "Precise computation of coaxial waveguide eigenvalues based on interlacing properties," in Conf. Proc. JINA, 2004.

[21] E. Sorolla and M. Mattes, "Globally convergent algorithm to find the zeros of the cross-product of Bessel functions," in Proc. Int. Conf. Electromagn. Adv. Appl., 2011, pp. 80-81.

[22] J. Segura, "A global Newton method for the zeros of cylinder functions," Numer. Alg., vol. 18, pp. 259-276, 1998.
[23] J. A. Cochran, "The analyticity of cross-product Bessel function zeros," Proc. Camb. Phil. Soc., vol. 62, pp. 215-226, 1966.

[24] W. H. Press, S. A. Teukolsky, W. T. Vetterling, and B. P. Flannery, Numerical Recipes in Fortran 77 (The Art of Scientific Computing), 2nd ed. Cambridge, U.K.: Cambridge Univ. Press, 1992, vol. 1.

[25] A. Gray and G. B. Mathews, A Treatise on Bessel Functions and their Applications to Physics. London, U.K.: MacMillan, 1895.

[26] M. Abramowitz and I. A. Stegun, Handbook of Mathematical Functions. New York, NY, USA: Dover, 1970.

[27] R. Truell, "Concerning the roots of $J_{\nu}^{\prime}(x) N_{\nu}^{\prime}(q x)-J_{\nu}^{\prime}(q x) N_{\nu}^{\prime}(x)=$ 0," J. Appl. Phys., vol. 14, pp. 350-352, 1943.

[28] H. B. Dwight, "Table of roots for natural frequencies in coaxial-type cavities," J. Math. Phys., vol. 27, pp. 84-89, 1948.

[29] M. Kline, "Some Bessel equations and their application to guide and cavity theory," J. Math. Phys., vol. 27, pp. 37-48, 1948.

[30] J. A. Ruiz-Cruz and J. M. Rebollar, "Eigenmodes of waveguides using a boundary contour mode-matching method with an FFT scheme," Int. J. RF Microw. Comput.-Aided Eng., vol. 15, no. 3, pp. 286-295, 2004.

[31] V. de la Rubia and J. Zapata, "An efficient method for determining TE and TM modes in closed waveguides made up of $\mathrm{N}$ cylindrical conductors," IEEE Trans. Microw. Theory Tech., vol. 53, no. 2, pp. 670-678, Feb. 2005.

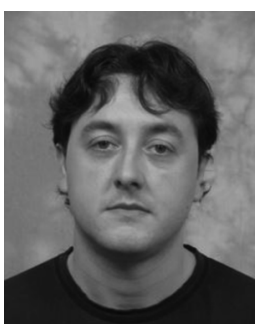

Edén Sorolla was born in Alicante, Spain, in 1980. He received the Physics degree from the Universidad de Valencia, Valencia, Spain in 2007.

From 2007 to 2008, he obtained a scholarship from the Departamento de Física Aplicada y Electromagnetismo and Instituto de Ciencia de Materiales, Universidad de Valencia, to study several electron sources and multipactor detection methods as well as the secondary-electron-yield properties of some materials. In 2008, he enrolled the Ph.D. studies at the Laboratory of Electromagnetics and Acoustics (LEMA), Ecole Polytechique Fédérale de Lausanne (EPFL), Lausanne, Switzerland, where he is currently a Student Assistant. He has been responsible for several European Space Agency (ESA) projects and a Swiss National Science Foundation (SNSF) project. His current research interest includes nonlinear electromagnetic phenomena appearing in power microwave subsystems (multipactor effect, corona effects, and passive intermodulation phenomena).

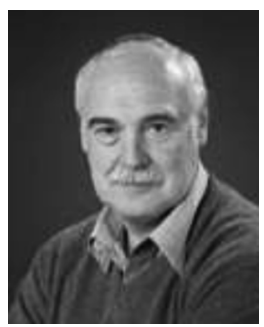

Juan R. Mosig (S'76-M'87-SM'94-F'99) was born in Cadiz, Spain. He received the Electrical Engineer degree from the Universidad Politécnica de Madrid, Madrid, Spain, in 1973, and the Ph.D. degree from Ecole Polytechnique Fédérale de Lausanne (EPFL), Lausanne, Switzerland, in 1983.

In 1976, he joined the Laboratory of Electromagnetics and Acoustics (LEMA), EPFL, of which he has been the Head since 2000. Since 1991, he has been a Professor with EPFL. In 1984, he was a Visiting Research Associate with the Rochester Institute of Technology, Rochester, NY, USA. He has also held scientific appointments with the University of Rennes, Rennes, France; University of Nice, Nice, France; Technical University of Denmark, Kongens Lyngby, Denmark; and University of Colorado at Boulder, Boulder, CO, USA. He has authored four chapters in books on microstrip antennas and circuits and over 120 reviewed papers. His research interests include electromagnetic theory, numerical methods, and planar antennas.

Dr. Mosig has been the Swiss Delegate for European COST Antenna Actions since the 1980's and the Chair for the two last COST Antenna Actions 284 and IC0603 ASSIST (2003-2011). He is also a founding member and General Chair of the European Association on Antennas and Propagation (EurAAP), owner of the EuCAP conference series. He is the originator of a successful annual workshop, INTELECT, on computational electromagnetics

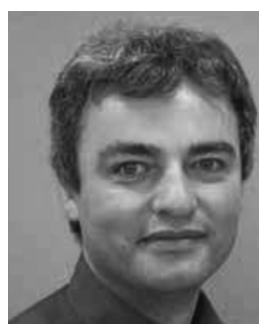

Michael Mattes (M'08) received the Diplom-Ingenieur degree from the University of Ulm, Ulm, Germany, in 1996, and the Dr. ès Sci. degree from Ecole Polytechnique Fédérale de Lausanne (EPFL), Lausanne, Switzerland, in 2003.

After one year as a Research Fellow with the Department of Microwave Techniques, University of Ulm, he joined the Laboratory of Electromagnetics and Acoustics (LEMA), EPFL, in 1997. His current research interests include computational electromagnetics in the framework of high-power phenomena in microwave devices and bio-electromagnetic problems. 